\title{
The Risk Assessment in Low-Grade Gliomas: An Analysis of the European Organization for Research and Treatment of Cancer (EORTC) and the Radiation Therapy Oncology Group (RTOG) criteria
}

Andrea Lanese - Enrico Franceschi · Alba A. Brandes

Received: May 2, 2018 / Published online: July 16, 2018

(C) The Author(s) 2018
Diffuse low-grade gliomas (LGG) are rare tumors that affect young adult patients. The European Organization for Research and Treatment of Cancer (EORTC) and the Radiation Therapy Oncology Group (RTOG) have both developed their own clinical prognostic scores to assist clinicians in treatment decision-making. These criteria have been used to include patients in phase III studies. To date, it is unknown which is the best score to define the prognosis of LGG. Additionally, a pure clinical classification is probably not a sufficiently informative basis for choosing the proper treatment in different situations. A combined score with both clinical and molecular features will likely be indispensable.

Keywords: Comparison; European Organization for Research and Treatment of Cancer (EORTC); Low-grade glioma; Prognostic scores;

Enhanced digital content To view enhanced digital content for this article go to https://doi.org/10.6084/ m9.figshare.6754967.

A. Lanese · E. Franceschi · A. A. Brandes $(\square)$ Department of Medical Oncology, Bellaria Hospital, Azienda Unitaria Sanitaria Locale (USL), Bologna, Italy

e-mail: alba.brandes@yahoo.it
Risk assessment; Radiation Therapy Oncology Group (RTOG)

\section{COMMENTARY}

Diffuse low-grade gliomas (LGG) are a group of primary brain cancers that are mainly characterized by their slow growth and infiltrative pattern and histologically distinguishable by the presence of grade II astrocytomas and oligodendrogliomas. The global incidence is approximately $3-15 \%$ of all brain tumors $[1,2]$. Young adults aged 20-40 years are usually affected, and these individuals often present with a long history of neurological symptoms, with $80 \%$ of patients presenting with seizure; headache and motor deficits are less common. It is notable that some patients with LGG are asymptomatic and that the LGG are incidentally diagnosed during medical tests carried for other reasons [3].

The 2007 World Health Organization (WHO) classification stratified LGG by histological features, such as astrocytoma, oligodendroglioma and oligoastrocytoma, with a focus on the mitotic index, which is generally low in these tumors [4]. However, a low level of diagnostic reproducibility among pathologists has been observed over the past years, which has prevented the development of a standard approach to the diagnosis of LGG [5]. A revised version of 
the WHO classification was published in 2016, wherein the integration of the isocitrate dehydrogenase (IDH) mutation and $1 \mathrm{p} 19 \mathrm{q}$ co-deletion plays a central role in the diagnostic procedure [6]. This has led to a re-definition of past diagnoses of LGG. In this revised classification, a good prognosis for LGG is maintained, and a long survival can be achieved with the correct therapeutic strategy.

In the past, a "wait and see" strategy was the most typical approach following surgery. However, more recent trials have demonstrated that there is a survival benefit to combined treatment with radiotherapy and chemotherapy post-surgery.

The debate surrounding the standard therapeutic approach to LGG has resulted in the European Organization for Research and Treatment of Cancer (EORTC) and the Radiation Therapy Oncology Group (RTOG) each independently developing their own prognostic score. Both scores list several clinical risk factors and have the aim to help clinicians in the treatment decision-making process.

The EORTC score was developed in 2002 by Pignatti et al. [7] based on their retrospective analysis of two multicenter prospective randomized phase III trials on the role of radiotherapy in LGG (EORTC 22844-22845) [8, 9]. The study population included patients affected by grade II astrocytoma, oligodendroglioma and mixed oligoastrocytoma according to the 1979 WHO classification. These authors found that an age of $\geq 40$, histological findings of astrocytoma, presence of neurological deficits before surgery, tumor diameter of $\geq 6 \mathrm{~cm}$ and tumor crossing the midline were prognostic factors of LGG [7]. They then categorized patients either as low risk in presence of $0-2$ factors or high risk when 3-5 factors were present, and the overall survival was 7.80 vs 3.67 years for low- and high-risk patients, respectively [7].

The RTOG considers only two clinical factors: age of $\geq 40$ years and a sub-total resection of the tumor. These two criteria were included in a prospective randomized phase III conducted by the RTOG. Patients with residual tumor after surgery who were younger than 40 years old or those who were aged $\geq 40$ years were considered to be at high risk and included in the trial, while patients without these factors received only follow-up after surgery [10, 11]. The RTOG trial documented an improved overall survival (OS) and improved progressionfree survival (PFS) with radiotherapy followed by PCV (procarbazine, CCNU and vincristine) chemotherapy. Patients treated with the sequential approach had a median OS of 13.3 years and a 5 -year PFS rate of $51 \%$, compared to those who received radiotherapy alone (median OS 7.8 years, 5-year PFS 21\%) [11].

Notably, both scores include age of $\geq$ 40 years as a poor prognostic factor. The prognostic impact of older age has been well demonstrated in several retrospective trials $[12,13]$. In their retrospective analysis carried out in 1997, Lote et al. [12] documented a worsening survival of LGG patients with increasing age, with an OS of 226 months in the age group 0-19 years and an OS of 39 months in patients aged $>59$ years.

The prognostic role of extent of resection (EOR) still remains open to debate. A retrospective trial carried out in 2008 showed that patients with an EOR of $<90 \%$ had 5-year OS rates of $76 \%$ and 8 -year OS rates of $60 \%$; in comparison, patients with an EOR of $>90 \%$ had a 5- and 8-year OS of 97 and 91\%, respectively [14]. No statistically significant impact of resection on survival was found in the EORTC study [7], and the authors claimed that the EOR depends on tumor size rather than on EOR itself. Similarly, Shibamoto did not find any association between EOR and prognosis in a retrospective study [15]. Therefore, EOR as a prognostic factor is included only in the RTOG criteria-and not in the EORTC score. Although the RTOG criteria do not take tumor diameter into account, in the phase II observational trial on low-risk patients a tumor diameter of $>4 \mathrm{~cm}$ was associated with a worse prognosis [10].

Molecular status was not considered in either score (EORTC or RTOG) because it had not been included in the WHO classification adopted during the study enrollment period. However, in 2016, the EORTC 22033 randomized, openlabel phase III study compared temozolomide with radiotherapy in high-risk LGG patients (based on EORTC score) and found a longer PFS for patients with IDH mutant/1p19q non- 
codeleted tumors treated with radiotherapy rather than chemotherapy [hazard ratio 1.86, $95 \%$ confidence interval 1.21-2.87, $p=0.0043$ ), but similar outcomes for those harboring IDH mutant/1p19q codeletion as well as IDH wildtype LGG [16]. It would appear, therefore, that the lack of molecular data makes both scores weak and incomplete.

Interestingly, a retrospective analysis by Wijnenga et al. [17] showed that the role of postoperative volume residual disease after surgery was particularly strong in IDH-mutated astrocytoma patients, where even very small postoperative volumes $\left(0.1-5.0 \mathrm{~cm}^{3}\right)$ negatively affected survival.

In conclusion, EORTC and RTOG criteria were developed to predict the progress of patients with LGG, with the overall aim of driving the treatment choice made by clinicians. However, the multicenter retrospective EORTC analysis and the RTOG randomized phase III trials that enrolled LGG patients according to these two different criteria are not comparable. Moreover, no data are available can support one set of criteria over the other. This situation highlights the need of a comparison between EORTC and RTOG criteria. However, it is important to remember that a pure clinical classification is likely not sufficiently informative to choose the proper treatment for different situations, and that a combined score which takes both clinical and molecular factors into account is likely to be indispensable.

\section{ACKNOWLEDGEMENTS}

The authors thank the patients and their families.

Funding. No funding or sponsorship was received for this study or publication of this article.

Authorship. All named authors meet the International Committee of Medical Journal Editors (ICMJE) criteria for authorship for this article, take responsibility for the integrity of the work as a whole, and have given their approval for this version to be published.

Disclosures. Andrea Lanese, Enrico Franceschi and Alba A Brandes have nothing to disclose.

Compliance with Ethics Guidelines. This article is based on previously conducted studies and does not contain any studies with human participants or animals performed by any of the authors.

Open Access. This article is distributed under the terms of the Creative Commons Attribution-NonCommercial 4.0 International License (http://creativecommons.org/licenses/ by-nc/4.0/), which permits any noncommercial use, distribution, and reproduction in any medium, provided you give appropriate credit to the original author(s) and the source, provide a link to the Creative Commons license, and indicate if changes were made.

\section{REFERENCES}

1. Rees J, Watt H, Jäger HR, et al. Volumes and growth rates of untreated adult low-grade gliomas indicate risk of early malignant transformation. Eur J Radiol. 2009;72(1):54-64.

2. Sahm F, Capper D, Jeibmann A, et al. Addressing diffuse glioma as a systemic brain disease with single-cell analysis. Arch Neurol. 2012;69(4):523-6.

3. Pouratian N, Asthagiri A, Jagannathan J, et al. Surgery insight: the role of surgery in the management of low-grade gliomas. Nat Clin Pract Neurol. 2007;3:628-39.

4. Louis DN, Ohgaki H, Wiestler OD, et al. The 2007 WHO classification of tumours of the central nervous system. Acta Neuropathol. 2007;114(2) :97-109.

5. Coons SW, Johnson PC, Scheithauer BW et al. Improving diagnostic accurancy and interobserver concordance in the classification and grading of primary gliomas. Cancer. 1997;79(7):1381-93.

6. Louis DN, Perry A, Reifenberger G, et al. The 2016 World Health Organization classification of tumors 
of the central nervous system: a summary. Acta Neuropathol. 2016;131:803-20.

7. Pignatti F, van den Bent M, Curran D, et al. Prognostic factors for survival in adult patients with cerebral low-grade glioma. J Clin Oncol. 2002;20(8):2076-84.

8. Karim ABMF, Maat B, Hatlevoll R, et al. A randomized trial on dose-response in radiation therapy of low grade cerebral glioma: European Organization for Research and Treatment of Cancer (EORTC) study 22844. Int J Radiat Oncol Biol Phys. 1996;36:549-56.

9. Karim ABMF, Afra D, Cornu P. Randomized trial of the efficacy of radiotherapy for low-grade glioma in the adult: European Organization for Research and Treatment of Cancer Study 22845 with the Medical Research Council study BR04: an interim analysis. Int J Radiat Oncol Biol Phys. 2002;52:316-24.

10. Shaw EG, Berkey B, Coons SW, et al. Recurrence following neurosurgeon-determined gross-total resection of adult supratentorial low-grade glioma: results of a prospective clinical trial. J Neurosurg. 2008;109(5):835-41.

11. Buckner JC, Shaw EG, Pugh SL, et al. Radiation plus procarbazine, CCNU, and vincristine in low-grade glioma. N Engl J Med. 2016;374(14):1344-55.

12. Lote K, Egeland T, Hager B, et al. Survival, prognostic factors, and therapeutic efficacy in low-grade glioma: a restrospective study in 379 patients. J Clin Oncol. 1997;15:3129-40.

13. Leighton C, Fisher B, Bauman G et al. Supratentorial low-grade glioma in adults: an analysis of prognostic factors and the timing of radiation. J Clin Oncol. 1997;15:1294-301.

14. Smith JS, Chang EF, Lamborn KR et al. Role of extent of resection in the long term outcome of low-grade hemispheric glioma. J Clin Oncol. 2008;26:1338-45.

15. Shibamoto Y, Kitakabu Y, Takahashi M et al. Supratentorial low-grade astrocytoma. Correlation of computed tomography findings with effect of radiation therapy and prognostic variables. Cancer. 1993;72(1):190-5.

16. Baumert BG, Hegi ME, van den Bent MJ et al. Temozolomide chemotherapy versus radiotherapy in highrisk low-grade glioma (EORTC 22033-26033): a randomised, open-label, phase 3 intergroup study. Lancet Oncol. 2016;17(11):1521-32.

17. Wijnenga MMJ, French PJ, Dubbink HJ, et al. The impact of surgery in molecularly defined low-grade glioma: an integrated clinical, radiological, and molecular analysis. Neuro Oncol. 2018;20(1):103-12. 